\title{
THE LINEARISATIONS OF CYCLIC PERMUTATIONS HAVE RATIONAL ZETA FUNCTIONS
}

\author{
BAU-SEN DU
}

\begin{abstract}
Let $n \geqslant 2$ be an integer. Let $P$ be the set of all integers in $[1, n+1]$ and let $\sigma$ be a cyclic permutation on $P$. Assume that $f$ is the linearisation of $\sigma$ on $P$. Then we show that $f$ has rational Artin-Mazur zeta function which is closely related to the characteristic polynomial of some $n \times n$ matrix with entries either zero or one. Some examples of non-conjugate maps with the same Artin-Mazur zeta function are also given.
\end{abstract}

Let $[a, b]$ be a nondegenerate compact interval on the real line and let $f$ be a continuous map from $[a, b]$ into itself. For every positive integer $k$, let $f^{k}$, the $k^{\text {th }}$ iterate of $f$, be defined by: $f^{1}=f$ and $f^{k}=f \circ f^{k-1}$ if $k>1$. For $x_{0} \in[a, b]$, we call $x_{0}$ a periodic point of $f$ if $f^{m}\left(x_{0}\right)=x_{0}$ for some positive integer $m$ and call the smallest such positive integer $m$ the least period of $x_{0}$ (under $f$ ). We call the set $\left\{f^{k}\left(x_{0}\right) \mid k\right.$ is any nonnegative integer $\}$ the periodic orbit of $x_{0}$ (under $f$ ). It is easy to see that, if $f^{m}\left(x_{0}\right)=x_{0}$ for some positive integer $m$, then the least period of $x_{0}$ must divide $m$. We shall need this fact later. A periodic point of least period 1 is also called a fixed point. In discrete dynamical systems theory, one problem related to the numbers of periodic points is: For every positive integer $k$, let $P_{k}=\left\{x \in[a, b] \mid f^{k}(x)=x\right\}$. Let $N\left(f^{k}\right)$ be the number of points in $\left\{x \in P_{k} \mid x\right.$ is isolated in $P_{m}$ for some positive integer $m$ dividing $\left.k\right\}$. Assume that, for every positive integer $k$, the number $N\left(f^{k}\right)$ is finite. (Note that this definition of $N\left(f^{k}\right)$ is a slight generalisation of that of Artin and Mazur [1].) Find the reduced Artin-Mazur zeta function [5] $\bar{\zeta}_{f}(z)=\sum_{k=1}^{\infty} N\left(f^{k}\right) z^{k}$ of $f$ or find the Artin-Mazur zeta function $[1,5] \zeta_{f}(z)=\exp \left(\sum_{k=1}^{\infty}\left(N\left(f^{k}\right) / k\right) z^{k}\right)$ of $f$, where $z$ is the complex variable. When we actually compute $[2,3]$ the reduced Artin-Mazur zeta functions of some special types of continuous piecewise linear maps $f$ on $[a, b]$, we find some $n \times n$ matrices $A_{f}$ (depending on $f$ ) such that $N\left(f^{k}\right)=\operatorname{tr}\left(A_{f}^{k}\right)$, the trace of $A_{f}^{k}$, for every positive integer $k$ (see also [6]) and the reduced Artin-Mazur zeta functions of $f$ are closely

Received 12th January, 2000

Copyright Clearance Centre, Inc. Serial-fee code: 0004-9727/00 \$A2.00+0.00. 
related to the characteristic polynomials of the matrices $A_{f}$. In this note, we extend this result to a class called the linearisations of cyclic permutations. To this end, we shall need the following result from matrix theory.

TheOREM 1 . Let $A$ be an $n \times n$ complex matrix and let $\operatorname{det}\left(x E_{n}-A\right)=x^{n}+$ $\sum_{j=0}^{n-1} \beta_{j} x^{j}$ be the characteristic polynomial of $A$, where $E_{n}$ is the $n \times n$ identity matrix. Then

$\sum_{k=1}^{\infty} \operatorname{tr}\left(A^{k}\right) z^{k}=-z \frac{\frac{d}{d z}\left(1+\sum_{j=1}^{n} \beta_{n-j} z^{j}\right)}{1+\sum_{j=1}^{n} \beta_{n-j} z^{j}}$ or $\exp \left(\sum_{k=1}^{\infty} \frac{\operatorname{tr}\left(A^{k}\right)}{k} z^{k}\right)=\frac{1}{1+\sum_{j=1}^{n} \beta_{n-j} z^{j}}$

ProOF: Write $z^{n}+\sum_{j=0}^{n-1} \beta_{j} z^{j}=\prod_{j=1}^{n}\left(z-\lambda_{j}\right)$. Then, by replacing $z$ by $1 / z$ and simplifying, we obtain that $1+\sum_{j=1}^{n} \beta_{n-j} z^{j}=\prod_{j=1}^{n}\left(1-\lambda_{j} z\right)$. Since $A$ is similar to an upper triangular matrix with main diagonal entries the eigenvalues of $A$, we easily obtain that $\operatorname{tr}\left(A^{k}\right)=\sum_{j=1}^{n} \lambda_{j}^{k}$ for all positive integers $k$. This fact will also be used later. So,

$$
\begin{aligned}
\sum_{k=1}^{\infty} \operatorname{tr}\left(A^{k}\right) z^{k} & =\sum_{k=1}^{\infty}\left(\sum_{j=1}^{n} \lambda_{j}^{k}\right) z^{k}=\sum_{j=1}^{n}\left(\sum_{k=1}^{\infty}\left(\lambda_{j} z\right)^{k}\right)=\sum_{j=1}^{n} \frac{\lambda_{j} z}{1-\lambda_{j} z} \\
& =-z \frac{\frac{d}{d z}\left(\prod_{j=1}^{n}\left(1-\lambda_{j} z\right)\right)}{\prod_{j=1}^{n}\left(1-\lambda_{j} z\right)}=-z \frac{\frac{d}{d z}\left(1+\sum_{j=1}^{n} \beta_{n-j} z^{j}\right)}{1+\sum_{j=1}^{n} \beta_{n-j} z^{j}}
\end{aligned}
$$

Or, by formal integration,

$$
\exp \left(\sum_{k=1}^{\infty} \frac{\operatorname{tr}\left(A^{k}\right)}{k} z^{k}\right)=\frac{1}{1+\sum_{j=1}^{n} \beta_{n-j} z^{j}}
$$

We now return to the discrete dynamical systems theory on the interval. Let $n \geqslant 2$ be an integer. Let $P$ be the set of all integers in $[1, n+1]$ and let $\sigma$ be a map from $P$ into itself. For every integer $1 \leqslant k \leqslant n$, let $J_{k}=[k, k+1]$. Assume that $f$ is the continuous map from $[1, n+1]$ into itself such that $f(i)=\sigma(i)$ for every integer $1 \leqslant i \leqslant n+1$ and $f$ is linear on $J_{k}$ (and so, the absolute value of the slope of $f$ on $J_{k}$ is $\geqslant 1$ ) for every integer $1 \leqslant k \leqslant n$. This continuous map $f$ is called the linearisation 
of $\sigma$ on $P$. Let $A_{f}=\left(\alpha_{i j}\right)$ be the $n \times n$ matrix defined by $\alpha_{i j}=1$ if $f\left(J_{i}\right) \supset J_{j}$ and $\alpha_{i j}=0$ otherwise. This matrix $A_{f}$ is called the Markov matrix of $f$. Note that, for every positive integer $k$, since $f$ is piecewise linear, so is $f^{k}$ and the slope of every linear piece of $f^{k}$ is the product of the slopes of $k$ linear pieces of $f$. Now, for every positive integer $k$, if $\alpha_{i_{1} i_{2}} \alpha_{i_{2} i_{3}} \cdots \alpha_{i_{k} i_{k+1}}$ is nonzero (in this case, this product is 1) for some integers $i_{1}, i_{2}, \cdots, i_{k+1}$ in $[1, n]$, then, by definition of $A_{f}, f\left(J_{i_{s}}\right) \supset J_{i_{s+1}}$ for every integer $1 \leqslant s \leqslant k$. If $I$ and $J$ are closed subintervals of $[1, n+1]$ such that $f(I) \supset J$, then it is well-known that there is a closed interval $L \subset I$ such that $f(L)=J$. So, since $f\left(J_{i_{k}}\right) \supset J_{i_{k+1}}$, there is a closed interval $L_{1} \subset J_{i_{k}}$ such that $f\left(L_{1}\right)=J_{i_{k+1}}$. Since $L_{1} \subset J_{i_{k}} \subset f\left(J_{i_{k-1}}\right)$, there is a closed interval $L_{2} \subset J_{i_{k-1}}$ such that $f\left(L_{2}\right)=L_{1}$. Inductively, there are closed intervals $L_{1}, L_{2}, \cdots, L_{k}$ such that $L_{s} \subset J_{i_{k+1-s}}$ and $f\left(L_{s}\right)=L_{s-1}$ for every integer $1 \leqslant s \leqslant k$, where we define $L_{0}=J_{i_{k+1}}$. Consequently, $f^{k}$ is linear on $L_{k} \subset J_{i_{1}}$ and $f^{k}\left(L_{k}\right)=J_{i_{k+1}}$. Note that, since $f^{k}$ is linear on $L_{k}$ and $f^{k}\left(L_{k}\right)=J_{i_{k+1}}$, the slope of $f^{k}$ on $L_{k}$ is nonzero.

Conversely, let $k$ be a fixed positive integer and let $T_{k}$ be a maximum closed interval on which $f^{k}$ is linear with nonzero slope. Since the $y$-coordinates of the turning points and the boundary points, $(1, f(1))$ and $(n+1, f(n+1))$, of $f$ are contained in $P, f^{k}\left(T_{k}\right)$ is a compact interval whose endpoints are distinct and contained in $P$. So, for some integers $1 \leqslant u_{k} \leqslant v_{k} \leqslant n, f^{k}\left(T_{k}\right)=\bigcup_{i=u_{k}}^{v_{k}} J_{i}$. Let $i_{k+1}$ be any integer such that $u_{k} \leqslant i_{k+1} \leqslant v_{k}$. Then $f^{k}\left(T_{k}\right) \supset J_{i_{k+1}}$ and there is a closed subinterval $L_{k}$ of $T_{k}$ such that $f^{k}\left(L_{k}\right)=J_{i_{k+1}}$. Since $f^{k}$ is linear on $L_{k}, f$ is linear on the interval $f^{s}\left(L_{k}\right)$ for every integer $0 \leqslant s \leqslant k-1$. In particular, $f$ is linear on the interval $f^{k-1}\left(L_{k}\right)$ and since the interior of $f\left(f^{k-1}\left(L_{k}\right)\right)=J_{i_{k+1}}$ contains no point of $P$, neither does the interior of $f^{k-1}\left(L_{k}\right)$. This implies that $f^{k-1}\left(L_{k}\right) \subset J_{i_{k}}$ for some integer $1 \leqslant i_{k} \leqslant n$. Similarly, since $f$ is linear on the interval $f^{k-2}\left(L_{k}\right)$ and the interior of $f\left(f^{k-2}\left(L_{k}\right)\right)=f^{k-1}\left(L_{k}\right) \subset J_{i_{k}}$ contains no point of $P$, neither does the interior of $f^{k-2}\left(L_{k}\right)$. Therefore, $f^{k-2}\left(L_{k}\right) \subset J_{i_{k-1}}$ for some integer $1 \leqslant i_{k-1} \leqslant n$. Similar arguments imply that there are integers $i_{k}, i_{k-1}, \cdots, i_{3}, i_{2}, i_{1}$ in $[1, n]$ such that $f^{t}\left(L_{k}\right) \subset J_{i_{t+1}}$ for every integer $0 \leqslant t \leqslant k-1$ and $f^{k}\left(L_{k}\right)=J_{i_{k+1}}$. Note that, when $t=0, L_{k} \subset J_{i_{1}}$. Thus, $f^{s}\left(L_{k}\right) \subset f\left(J_{i_{s}}\right)$ for every integer $1 \leqslant s \leqslant k$. Consequently, $f^{s}\left(L_{k}\right) \subset J_{i_{s+1}} \cap f\left(J_{i_{s}}\right)$ for every integer $1 \leqslant s \leqslant k-1$. So, $f\left(J_{i_{s}}\right)$ contains some interior points of $J_{i_{s+1}}$ for every integer $1 \leqslant s \leqslant k-1$. Since both endpoints of $f\left(J_{i_{s}}\right)\left(\supset f^{s}\left(L_{k}\right)\right)$ are distinct points in $P$, this implies that $f\left(J_{i_{s}}\right) \supset J_{i_{s+1}}$, and so, $\alpha_{i_{s} i_{s+1}}=1$ for every integer $1 \leqslant s \leqslant k-1$. Furthermore, since $J_{i_{k}} \supset f^{k-1}\left(L_{k}\right)$, we have $f\left(J_{i_{k}}\right) \supset f\left(f^{k-1}\left(L_{k}\right)\right)=J_{i_{k+1}}$. Thus, $\alpha_{i_{k} i_{k+1}}=1$. Hence $\alpha_{i_{1} i_{2}} \alpha_{i_{2} i_{3}} \cdots \alpha_{i_{k} i_{k+1}}=1$. Therefore, for every positive integer $k$, there is a one-to-one correspondence between the collection of nonzero products $\alpha_{i_{1} i_{2}} \alpha_{i_{2} i_{3}} \cdots \alpha_{i_{k} i_{k+1}}$ and the collection of closed 
intervals $L_{k} \subset J_{i_{1}}$ such that $f^{k}$ is linear on $L_{k}$ and $f^{k}\left(L_{k}\right)=J_{i_{k+1}}$. Consequently, if $A_{f}^{k}=\left(\alpha_{i j}^{(k)}\right)$; then every entry $\alpha_{i j}^{(k)}$ represents the number of closed intervals $L_{k} \subset J_{i}$ with disjoint interiors such that $f^{k}$ is linear on $L_{k}$ and $f^{k}\left(L_{k}\right)=J_{j}$. In the following, we shall show how to relate this number $\sum_{j=1}^{n} \alpha_{j j}^{(k)}$ to the number $N\left(f^{k}\right)$. We have two cases to consider:

CASE 1. Assume that, for some positive integer $t$, the absolute value of the slope of every linear piece of $f^{t}$ is $>1$. Then, it is easy to see that, for every integer $j>t$, the absolute value of the slope of every linear piece of $f^{j}$ is $>1$. So, for any integers $1 \leqslant i \leqslant n, k \geqslant 1$ (we do not require $k \geqslant t$ ) and any closed interval $L \subset J_{i}$, if $f^{k}$ is linear on $L$ and $f^{k}(L)=J_{i} \supset L$, then the slope of $f^{k}$ on $L$ cannot be equal to 1 and hence the equation $f^{k}(x)=x$ has exactly one solution in $L$. So, we can associate this unique solution to the interval $L$. This implies that, for every positive integer $k$, $N\left(f^{k}\right)=\sum_{j=1}^{n} \alpha_{j j}^{(k)}=\operatorname{tr}\left(A_{f}^{k}\right)=\sum_{j=1}^{n} \lambda_{j}^{k}$, where $\lambda_{1}, \lambda_{2}, \cdots, \lambda_{n}$ are the eigenvalues of the Markov matrix $A_{f}$ of $f$.

CASE 2. Assume that the absolute value of the slope of some linear piece of $f^{n}$ is 1 . Let $T_{n}$ be a maximum closed interval on which $f^{n}$ is linear and the absolute value of the slope of $f^{n}$ on $T_{n}$ is 1 . As was just shown above, there exist integers $i_{1}, i_{2}, \cdots, i_{n+1}$ in $[1, n]$ and a closed interval $L_{n} \subset T_{n}$ such that $J_{i_{n+1}} \subset f^{n}\left(T_{n}\right), f^{n}\left(L_{n}\right)=J_{i_{n+1}}$ and $f^{s}\left(L_{n}\right) \subset J_{i_{s+1}}$ for every integer $0 \leqslant s \leqslant n-1$. Since the absolute value of the slope of $f^{n}$ on $L_{n}$ (which is 1 ) equals the product of the absolute values of the slopes of $f$ on $f^{s}\left(L_{n}\right)$ (which are $\geqslant 1$ ) for all integers $0 \leqslant s \leqslant n-1$, the absolute value of the slope of $f$ on $f^{s}\left(L_{n}\right)$ must be 1 for every integer $0 \leqslant s \leqslant n-1$. So, the length of $f^{s}\left(L_{n}\right)$ is equal to that of $f^{s+1}\left(L_{n}\right)$ for every integer $0 \leqslant s \leqslant n-1$. Since the length of $f^{n}\left(L_{n}\right)\left(=J_{i_{n+1}}\right)$ is 1 , we obtain that the length of $f^{s}\left(L_{n}\right)\left(\subset J_{i_{s+1}}\right)$ is also 1 for every integer $0 \leqslant s \leqslant n-1$. This, together with the fact that $f^{n}\left(L_{n}\right)=J_{i_{n+1}}$, implies that $f^{s}\left(L_{n}\right)=J_{i_{s+1}}$ for every integer $0 \leqslant s \leqslant n$. Note that, when $s=0$, $L_{n}=J_{i_{1}}$. Thus, $f\left(J_{i_{s}}\right)=f\left(f^{s-1}\left(L_{n}\right)\right)=f^{s}\left(L_{n}\right)=J_{i_{s+1}}$ for every integer $1 \leqslant s \leqslant n$. Since there are $n+1$ closed intervals $J_{i_{s+1}}\left(=f^{s}\left(L_{n}\right)\right), 0 \leqslant s \leqslant n$, taken from the $n$ distinct $J_{i}$ 's, some interval $J_{i}$, appears at least twice. Without loss of generality, we may assume that $J_{i_{1}}=J_{i_{m+1}}$ for some integer $1 \leqslant m \leqslant n$ and the $J_{i_{s}}$ 's are distinct for all integers $1 \leqslant s \leqslant m$. Since $f\left(J_{i_{s}}\right)=J_{i_{s+1}}$ and $f$ is linear on $J_{i_{s}}$ for all integers $1 \leqslant s \leqslant m$, we obtain that, for every integer $1 \leqslant s \leqslant m, f^{m}\left(J_{i_{s}}\right)=J_{i_{s}}$ and $f^{m}$ is linear on $J_{i_{s}}$, and hence, we have either $f^{m}(x)=x$ for all $x \in J_{i_{s}}$ and all integers $1 \leqslant s \leqslant m$ or $f^{m}(x)=-x+a_{s}+b_{s}$, where $J_{i_{s}}=\left[a_{s}, b_{s}\right]$, for all $x \in J_{i s}$ and all integers $1 \leqslant s \leqslant m$. In the following, we assume, for simplicity, that $\sigma$ is a cyclic permutation on $P$. If, for some integer $1 \leqslant j \leqslant m, f^{m}(x)=x$ for all $x \in J_{i_{j}}$, then, in particular, 
$f^{m}\left(a_{j}\right)=a_{j}$. Since $a_{j}$ is a periodic point of $f$ with least period $n+1$, we must have $n+1 \leqslant m$ which contradicts the assumption that $1 \leqslant m \leqslant n$. This contradiction implies that $f^{m}(x)=-x+a_{s}+b_{s}$ and hence $f^{2 m}(x)=x$ for all $x \in J_{i_{s}}$ and all integers $1 \leqslant s \leqslant m$. In particular, $f^{2 m}\left(a_{s}\right)=a_{s}$ for all integers $1 \leqslant s \leqslant m$. Since the least period of $a_{s}$ is $n+1, n+1$ divides $2 m$. Thus, $2 m=r(n+1)$ for some positive integer $r$. But, since $1 \leqslant m \leqslant n$, we must have $r=1$, and so, $n+1=2 m$. Furthermore, since $f^{m}$ maps every endpoint of $J_{i_{s}}$ to the other for every integer $1 \leqslant s \leqslant m$, the $m$ closed intervals $J_{i_{s}}$ 's are pairwise disjoint. Since there are exactly $n=2 m-1=m+(m-1)$ distinct closed intervals $J_{i}$ 's, we obtain that $\left\{J_{i_{s}} \mid 1 \leqslant s \leqslant m\right\}=\left\{J_{2 j-1} \mid 1 \leqslant j \leqslant m\right\}$. Consequently, $f^{m}(x)=-x+4 s-1$ and $f^{2 m}(x)=x$ for all $x \in J_{2 s-1}$ and all integers $1 \leqslant s \leqslant m$. This also implies that $T_{n}=J_{2 j-1}$ for some integer $1 \leqslant j \leqslant m$ and the absolute value of the slope of every linear piece of $f^{n}$ (and hence of $f^{k}$ for every integer $k \geqslant n$ ) on any closed interval contained in $J_{2 i}$ for any integer $1 \leqslant i \leqslant m-1$ is $>1$. Thus, for any positive integer $k$ (we do not require $k \geqslant n$ ), any integer $1 \leqslant i \leqslant m-1$ and any closed interval $L \subset J_{2 i}$, if $f^{k}$ is linear on $L$ and $f^{k}(L)=L_{2 i} \supset L$, then the slope of $f^{k}$ on $L$ cannot be equal to 1 and hence the equation $f^{k}(x)=x$ has a unique solution in $L$. So, we can associate this unique solution to the interval $L$. Furthermore, since $f$ permutes cyclically the intervals $J_{2 j-1}, 1 \leqslant j \leqslant m$, and since $f^{m}$ maps every endpoint of any $J_{2 j-1}, 1 \leqslant j \leqslant m$, to the other, we obtain that there exists a cyclic permutation $\rho$ on the set of all integers in $[1, m]$ such that $\sigma^{j}(1) \in\left\{2 \rho^{j}(1)-1\right.$, $\left.2 \rho^{j}(1)\right\}$ and $\left\{\sigma^{j}(1), \sigma^{m+j}(1)\right\}=\left\{2 \rho^{j}(1)-1,2 \rho^{j}(1)\right\}$ for all integers $1 \leqslant j \leqslant m$. On the other hand, since $f$ permutes cyclically the intervals $J_{2 j-1}, 1 \leqslant j \leqslant m$, and since $f^{m}(x)=-x+4 j-1$ and $f^{2 m}(x)=x$ for all $x \in J_{2 j-1}$ and all integers $1 \leqslant j \leqslant m$, we see that, for any positive integer $k$, (i) if $k$ is not a multiple of $m$, then the equation $f^{k}(x)=x$ has no solution in $J_{2 j-1}$ for any integer $1 \leqslant j \leqslant m$; (ii) if $k$ is an odd multiple of $m$, then each midpoint of $J_{2 j-1}$ is the unique (and isolated) solution of the equation $f^{k}(x)=x$ in $J_{2 j-1}$ for every integer $1 \leqslant j \leqslant m$; and (iii) if $k$ is an even multiple of $m$, then, for every integer $1 \leqslant j \leqslant m$, every point of $J_{2 j-1}$ is a (nonisolated) solution of the equation $f^{k}(x)=x$, but, the midpoint of $J_{2 j-1}$ is an isolated solution of the equation $f^{m}(x)=x$. Therefore, we can associate, for every integer $1 \leqslant j \leqslant m$, the midpoint of $J_{2 j-1}$ to the interval $J_{2 j-1}$ when $k$ is an (odd or even) multiple of $m$ and nothing otherwise. This implies that, for every positive integer $k$, $N\left(f^{k}\right)=\sum_{j=1}^{n} \alpha_{j j}^{(k)}=\operatorname{tr}\left(A_{f}^{k}\right)=\sum_{j=1}^{n} \lambda_{j}^{k}$, where $\lambda_{1}, \lambda_{2}, \cdots, \lambda_{n}$ are the eigenvalues of the Markov matrix $A_{f}$ of $f$. Consequently, by Theorem 1, we have proved the following two results:

THEOREM 2. Let $n \geqslant 2$ be an integer. Let $P$ be the set of all integers in $[1, n+1]$ and let $\sigma$ be a map from $P$ into itself. Assume that $f$ is the linearisation of $\sigma$ on $P$ such that, for some positive integer $t$, the absolute value of the slope of every linear 
piece of $f^{t}$ is $>1$. Then the following hold:

(a) For every positive integer $k, N\left(f^{k}\right)=\operatorname{tr}\left(A_{f}^{k}\right)=\sum_{j=1}^{n} \lambda_{j}^{k}$, where $\lambda_{1}, \lambda_{2}, \cdots$, $\lambda_{n}$ are the eigenvalues of the Markov matrix $A_{f}$ of $f$.

(b) The Artin-Mazur zeta function $\zeta_{f}(z)$ of $f$ is $\zeta_{f}(z)=1 /\left(1+\sum_{k=1}^{n} \beta_{n-k} z^{k}\right)$, where $x^{n}+\sum_{k=0}^{n-1} \beta_{k} x^{k}$ is the characteristic polynomial of the Markov matrix of $f$.

THEOREM 3. Let $n \geqslant 2$ be an integer. Let $P$ be the set of all integers in $[1, n+1]$ and let $\sigma$ be a map from $P$ into itself. For every integer $1 \leqslant k \leqslant n$, let $J_{k}=[k, k+1]$. Assume that $f$ is the linearisation of $\sigma$ on $P$ such that the absolute value of the slope of some linear piece of $f^{n}$ is 1 . Then there exist an integer $1 \leqslant m \leqslant n$ and $m$ distinct integers $i_{1}, i_{2}, \cdots, i_{m}$ in $[1, n]$ such that $f$ is linear on $J_{i_{s}}$ and $f\left(J_{i_{s}}\right)=J_{i_{s+1}}$ for every integer $1 \leqslant s \leqslant m$, where we define $i_{m+1}=i_{1}$. Furthermore, if $\sigma$ is a cyclic permutation on $P$, then the following also hold:

(a) $n+1=2 m$.

(b) $f^{m}(x)=-x+4 k-1$ and $f^{2 m}(x)=x$ for all $x \in J_{2 k-1}$ and all integers $1 \leqslant k \leqslant m$. In particular, $f$ has periodic points of least period $(n+1) / 2$.

(c) There exists a cyclic permutation $\rho$ on the set of all integers in $[1, m]$ such that $\sigma^{j}(1) \in\left\{2 \rho^{j}(1)-1,2 \rho^{j}(1)\right\}$ and $\left\{\sigma^{j}(1), \sigma^{m+j}(1)\right\}=\left\{2 \rho^{j}(1)-\right.$ $\left.1,2 \rho^{j}(1)\right\}$ for all integers $1 \leqslant j \leqslant m$.

(d) For every positive integer $k, N\left(f^{k}\right)=\operatorname{tr}\left(A_{f}^{k}\right)=\sum_{j=1}^{n} \lambda_{j}^{k}$, where $\lambda_{1}, \lambda_{2}, \cdots$, $\lambda_{n}$ are the eigenvalues of the Markov matrix $A_{f}$ of $f$.

(e) The Artin-Mazur zeta function $\zeta_{f}(z)$ of $f$ is $\zeta_{f}(z)=1 /\left(1+\sum_{k=1}^{n} \beta_{n-k} z^{k}\right)$, where $x^{n}+\sum_{k=0}^{n-1} \beta_{k} x^{k}$ is the characteristic polynomial of the Markov matrix of $f$.

REMARK. We require $\sigma$ to be a cyclic permutation on $P$ in Theorem 3 while not in Theorem 2. This is because the requirement on the slope of $f$ in Theorem 3 is weaker than that in Theorem 2. If we do not make the stronger requirement on $\sigma$ in Theorem 3 , there would be many trivial examples whose linearisations have well-defined Markov matrices while their Artin-Mazur zeta functions are not defined.

The following partial converse of Theorem 3 is easy to prove.

THEOREM 4. Let $m$ and $n$ be positive integers such that $n+1=2 m$. Let $\rho$ be a cyclic permutation on the set of all integers in $[1, m]$ and let $\sigma$ be a cyclic permutation on the set $P$ of all integers in $[1, n+1]$. For every integer $1 \leqslant i \leqslant n$, let $J_{i}=[i, i+1]$. 
Assume that $f$ is the linearisation of $\sigma$ on $P$ and $\sigma^{j}(1) \in\left\{2 \rho^{j}(1)-1,2 \rho^{j}(1)\right\}$ and $\left\{\sigma^{j}(1), \sigma^{m+j}(1)\right\}=\left\{2 \rho^{j}(1)-1,2 \rho^{j}(1)\right\}$ for all integers $1 \leqslant j \leqslant m$. Then, for every positive integer $k$, the absolute value of the slope of $f^{k}$ on $J_{2 j-1}$ is 1 for all integers $1 \leqslant j \leqslant m$. Consequently, the Artin-Mazur zeta function $\zeta_{f}(z)$ of $f$ is $\zeta_{f}(z)=1 /\left(1+\sum_{k=1}^{n} \beta_{n-k} z^{k}\right)$, where $x^{n}+\sum_{k=0}^{n-1} \beta_{k} x^{k}$ is the characteristic polynomial of the Markov matrix of $f$.

REMARK. If $\sigma$ is a cyclic permutation on the set $P$ of all integers in $[1, n+1]$ and $f$ is the linearisation of $\sigma$ on $P$, then Theorems $2,3, \& 4$ give a complete solution of the Artin-Mazur zeta function of $f$. In particular, the Artin-Mazur zeta function of $f$ is rational with poles at the values $1 / \lambda_{j}$ where $\lambda_{1}, \lambda_{2}, \cdots, \lambda_{n}$ are the eigenvalues of the Markov matrix of $f$.

Let $f$ and $g$ be two continuous maps from $[a, b]$ into itself. If they are (topologically) conjugate to each other, then it is clear that they have the same (if defined) Artin-Mazur zeta function. However, if they are not conjugate to each other, they may still have the same Artin-Mazur zeta function. For example, assume that both $f$ and $g$ satisfy the conditions in Theorem 2 above. If their respective Markov matrices are similar to each other, then, since similar matrices have the same characteristic polynomial [4], they have, by Theorem 2, the same Artin-Mazur zeta function. In the following, we present some such examples. The following result is taken from [2].

THEOREM 5. For every integer $n \geqslant 3$, let $f_{n}(x)$ be the continuous map from $[1, n]$ onto itself defined by

$$
f_{n}(x)= \begin{cases}x+1, & \text { for } 1 \leqslant x \leqslant n-1 \\ -(n-1) x+n^{2}-n+1, & \text { for } n-1 \leqslant x \leqslant n\end{cases}
$$

We also define sequences $\left\langle b_{k, n}\right\rangle$ as follows:

$$
b_{k, n}= \begin{cases}2^{k}-1, & \text { for } 1 \leqslant k \leqslant n-1, \\ \sum_{i=1}^{n-1} b_{k-i, n}, & \text { for } n \leqslant k\end{cases}
$$

Then, for any integers $k \geqslant 1$ and $n \geqslant 3, b_{k, n}$ is the number of distinct fixed points of the map $f_{n}^{k}(x)$ in $[1, n]$. Moreover, the Artin-Mazur zeta function $\zeta_{f_{n}}(z)$ of $f_{n}$, for every integer $n \geqslant 3$, is $\zeta_{f_{n}}(z)=1 /\left(1-\sum_{k=1}^{n-1} z^{k}\right)$.

THEOREM 6. For every odd integer $m \geqslant 3$, let $g_{m}(x)$ and $h_{m}(x)$ be the contin- 
uous maps from $[1, m]$ onto itself defined by

$$
g_{m}(x)= \begin{cases}-x+m+1, & \text { for } 1 \leqslant x \leqslant \frac{1}{2}(m-1), \\ -\frac{1}{2}(m+1) x+\frac{1}{4}(m+1)^{2}+1, & \text { for } \frac{1}{2}(m-1) \leqslant x \leqslant \frac{1}{2}(m+1), \\ \frac{1}{2}(m-1) x-\frac{1}{4}\left(m^{2}-1\right)+1, & \text { for } \frac{1}{2}(m+1) \leqslant x \leqslant \frac{1}{2}(m+1)+1, \\ -x+m+2, & \text { for } \frac{1}{2}(m+1)+1 \leqslant x \leqslant m .\end{cases}
$$

and

$$
h_{m}(x)= \begin{cases}x+\frac{1}{2}(m-1), & \text { for } 1 \leqslant x \leqslant \frac{1}{2}(m+1), \\ -(m-1) x+\frac{1}{2}\left(m^{2}+2 m-1\right), & \text { for } \frac{1}{2}(m+1) \leqslant x \leqslant \frac{1}{2}(m+1)+1 \\ x-\frac{1}{2}(m+1), & \text { for } \frac{1}{2}(m+1)+1 \leqslant x \leqslant m .\end{cases}
$$

Then, for any odd integer $m \geqslant 3$, both $g_{m}(x)$ and $h_{m}(x)$ have the same Artin-Mazur zeta function as $f_{m}(x)$, where $f_{m}(x)$ is defined as in Theorem 5 above.

ProOf: Let $m \geqslant 3$ be an odd integer. It suffices to show that the Markov matrices of $f_{m}, g_{m}$, and $h_{m}$ are similar to one another. Indeed, let $P$ be the set of all integers in $[1, m]$ and let $\sigma$ be a cyclic permutation on $P$. Let $\varphi$ be the linearisation of $\sigma$ on $P$ and let $V_{m-1}$ be the vector space over the field of real numbers with the set $Q_{1}=\left\{J_{1}, J_{2}, \cdots, J_{m-1}\right\}$ as a basis, where, for every integer $1 \leqslant k \leqslant m-1$, $J_{k}=[k, k+1]$. Then, $\varphi$ determines a linear transformation (which we call $\bar{\varphi}$ ) on $V_{m-1}$ defined by $\bar{\varphi}\left(\sum_{k=1}^{m-1} r_{k} J_{k}\right)=\sum_{k=1}^{m-1} r_{k} \bar{\varphi}\left(J_{k}\right)$, where $r_{k}$ 's are real numbers and $\bar{\varphi}\left(J_{k}\right)=\sum_{s=i_{k}}^{j_{k}} J_{s}$ if $\varphi\left(J_{k}\right)=\bigcup_{s=i_{k}}^{j_{k}} J_{s}$ for some integers $1 \leqslant i_{k} \leqslant j_{k} \leqslant m-1$. Furthermore, with respect to the basis $Q_{1}$, the linear transformation $\bar{\varphi}$ is also determined [4] by the $(m-1) \times(m-1)$ matrix $B_{\varphi}=\left(\beta_{i j}\right)$ in such a way that, for every integer $1 \leqslant k \leqslant m-1, \bar{\varphi}\left(J_{k}\right)=\sum_{j=1}^{m-1} \beta_{k j} J_{j}\left(=\sum_{s=i_{k}}^{j_{k}} J_{s}\right)$ which happens to be the same as the Markov matrix of the map $\varphi$ on $[1, m]$. Now, if we take $Q_{2}=\left\{J_{1}, J_{m-1}, J_{2}, J_{m-2}, \cdots, J_{i}, J_{m-i}, \cdots, J_{(m-3) / 2}, J_{(m+3) / 2}, J_{(m-1) / 2}, \sum_{k=1}^{(m+1) / 2} J_{k}\right\}$ as a new basis for $V_{m-1}$, then it is easy to see that $\bar{g}_{m}$ acts on $Q_{2}$ like $\bar{f}_{m}$ on $Q_{1}$. Similarly, if we take $Q_{3}=\left\{J_{(m-1) / 2}, J_{m-1}, J_{(m-3) / 2}, J_{m-2}, J_{(m-5) / 2}, J_{m-3}, \ldots\right.$, $\left.J_{3}, J_{(m-1) / 2+3}, J_{2}, J_{(m-1) / 2+2}, J_{1}, J_{(m-1) / 2+1}\right\}$ as a new basis for $V_{m-1}$, then $\bar{h}_{m}$ acts 
on $Q_{3}$ like $\bar{f}_{m}$ on $Q_{1}$. Therefore, the matrices of the linear transformations $\bar{f}_{m}, \bar{g}_{m}$, and $\bar{h}_{m}$ on the respective bases $Q_{1}, Q_{2}$, and $Q_{3}$ are the same. So, the matrices of $\bar{f}_{m}, \bar{g}_{m}$, and $\bar{h}_{m}$ on the basis $Q_{1}$ are similar to one another [4]. Consequently, the Markov matrices of the maps $f_{m}, g_{m}$, and $h_{m}$ on the interval $[1, m]$ are similar to one another and hence, by Theorem $2, f_{m}, g_{m}$, and $h_{m}$ have the same Artin-Mazur zeta function.

\section{REFERENCES}

[1] M. Artin and B. Mazur, 'On periodic points', Ann. Math. 81 (1965), 82-99.

[2] B.-S. Du, 'A simple method which generates infinitely many congruence identities', $F i$ bonacci Quart. 27 (1989), 116-124.

[3] B.-S. Du, 'Congruence identities arising from dynamical systems', Appl. Math. Lett. 12 (1999), 115-119.

[4] I.N. Herstein, Topics in algebra, first edition (Blaisdell Publ. Co., New York, 1964).

[5] J. Milnor and W. Thurston, On iterated maps of the interval, Lecture Notes in Mathematics 1342 (Springer-Verlag, Berlin, Heidelberg, New York, 1988), pp. 465-563.

[6] H.E. Nusse, 'Chaotic maps with rational zeta function', Trans. Amer. Math. Soc. 304 (1987), 705-719.

Institute of Mathematics

Academia Sinica

Taipei

Taiwan 11529

Republic of China

e-mail: mabsdu@sinica.edu.tw 